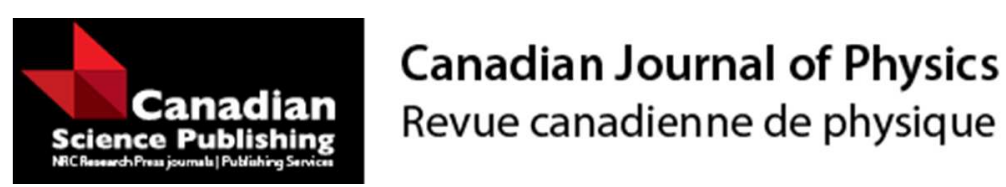

\title{
Atomic properties and transition parameters of highly charged Sb-like isoelectronic ions
}

\begin{tabular}{|r|l|}
\hline Journal: & Canadian Journal of Physics \\
\hline Manuscript ID & cjp-2016-0740.R2 \\
\hline Manuscript Type: & Article \\
\hline Date Submitted by the Author: & 19 -Jan-2017 \\
\hline Complete List of Authors: & $\begin{array}{l}\text { Huo, Xiaoxuan; Sichuan University } \\
\text { Jiang, Gang; Sichuan University } \\
\text { Li, Xiangfu; Sichuan University, }\end{array}$ \\
\hline Keyword: & $\begin{array}{l}\text { highly charged ions, atomic clocks, atomic properties, transition } \\
\text { parameters, Sb-like isoelectronic ions }\end{array}$ \\
\hline
\end{tabular}

\section{SCHOLARONE ${ }^{\text {Tw }}$ \\ Manuscripts}




\title{
Atomic properties and transition parameters of highly charged Sb-like isoelectronic ions
}

\author{
Xiaoxuan Huo, Gang Jiang ${ }^{*}$, Xiangfu Li \\ Institute of Atomic and Molecular Physics, Sichuan University, Chengdu 610065, Sichuan, \\ People's Republic of China
}

\begin{abstract}
The atomic properties and transition parameters of Sb-like $\mathrm{Cs}^{4+}, \mathrm{Ba}^{5+}, \mathrm{La}^{6+}, \mathrm{Ce}^{7+}, \mathrm{Pr}^{8+}$, $\mathrm{Nd}^{9+}, \mathrm{Pm}^{10+}$ and $\mathrm{Sm}^{11+}$ ions were first calculated using the GRASP2K package. This method is based on the multi-configuration Dirac-Hartree-Fock (MCDHF) method. In this calculation, the contributions of Breit and QED corrections were taken into consideration. By comparison, our results were in good agreement with the NIST values for $\mathrm{Cs}^{4+}$ and $\mathrm{Ba}^{5+}$. It revealed that our calculations are dependable. Meanwhile, it also showed that these ions have high sensitivity to the fine-structure constant $\alpha$ and long-lived metastable states. Especially $\mathrm{Pm}^{10+}$ and $\mathrm{Sm}^{11+}$ had very long-lived metastable states with transitions within the optical transition. These ions are useful for the research of ultra-accurate atomic clocks and fundamental physics.
\end{abstract}

\section{Introduction}

In recent years, the great progress has been made in the study of the accuracy and stability of optical frequency standards. The most precise study in experiment was $5.2 \times 10^{-17}$ from the frequence ratio of $\mathrm{AL}^{+}$and $\mathrm{Hg}^{+}$optical atomic clocks [1]. The most accurate trapped-ion clocks were two $\mathrm{Al}^{+}$clocks which have the fractional frequency uncertainty of $8.6 \times 10^{-18}$ [2], the accuracy of an optical lattice clock based on $\mathrm{Sr}$ atoms has been reported with an inaccuracy of $1.5 \times 10^{-16}$ [3]. The more accurate frequency standard is essential for further study of the fine-structure constant $\alpha$. Recent researches have found that the fractional uncertainty of the transition rate in the atomic clocks which based on highly charged ions (HCIs) can be smaller than $10^{-19}$, the sensitivity to the variation of $\alpha$ is assessed as up to $10^{-20}$ year $^{-1}[4,5]$. Hence the highly charged ions can be the candidates for atomic clocks, search for variation of fine-structure constant, and quantum information. These ions must have the optical transition so that they can be excited by available lasers. And they should have metastable states with long lifetimes so that they can be made atomic clocks. Therefore it is necessary to study the energy levels, transition rates and lifetimes of these ions [6]. And furthermore, the research about Sb-isoelectronic sequence ions are lacking and our research can add the database and offer a reference. Meanwhile, the Sb-like ions belong to multiple electron system and our results can provide a reference for other scientific research in this respect.

There were many scholars and research institutes have made studies on this subject. M. S. Safronova and S. G. Porsev et al. have researched it in depth, they studied atomic properties of Cd-like and Sn-like ions for the development of 
frequency standards and researched the variation of the fine-structure constant in 2014 [7]. In 2014, they studied the energies, transition wavelengths and lifetimes of Ag-like and In-like ions [6]. J. C. Berengut and V. A. Dzuba investigated an optical frequency standard according to the electric-octupole reference transition in a single trapped ${ }^{171} \mathrm{Yb}^{+}$ion in experiment in 2012 [8].

There are studies found out that transitions near level crossings in HCIs can provide higher sensitivity to $\alpha$ variation than any other optical transitions seen in atomic systems $[9,10]$. The level crossing refers to the energies of some orbitals which have larger principal quantum number are smaller than orbitals which have smaller principal quantum number, it is because that the filling order of electron shells in neutral atoms depends on the Madelung rule but changes to the Coulomb rule with the increasing of ion charge, Zion. Most level crossings occur at a relatively low ion stage, the interesting cases are those that occur in HCIs with $Z_{\text {ion }} \geqslant 5$, the crossing of $4 f-5$ s occurs at a relatively higher degree of ionization [11].

In this work, we calculated the atomic properties and transition parameters of Sb-like $\mathrm{Cs}^{4+}, \mathrm{Ba}^{5+}, \mathrm{La}^{6+}, \mathrm{Ce}^{7+}, \mathrm{Pr}^{8+}, \mathrm{Nd}^{9+}, \mathrm{Pm}^{10+}$ and $\mathrm{Sm}^{11+}$ ions using the GRASP2K package. This package is based on the multi-configuration Dirac-Hartree-Fock (MCDHF) method. We analyzed the level crossing and transition parameters of these ions in section 3. Breit and quantum electrodynamic (QED) corrections were included into the energies. Because we were focused on the ions near level crossing, we just discussed the transition rates and lifetimes of $\mathrm{La}^{6+}, \mathrm{Ce}^{7+}, \mathrm{Pr}^{8+}, \mathrm{Nd}^{9+}, \mathrm{Pm}^{10+}$ and $\mathrm{Sm}^{11+}$ ions. There are just Sb-like $\mathrm{Cs}^{4+}$ and $\mathrm{Ba}^{5+}$ ions which have been studied before and we can examine the accuracy of our study by compared the results of $\mathrm{Cs}^{4+}$ and $\mathrm{Ba}^{5+}$ with NIST [12].

\section{Method}

In our work, the package of GRASP2K was used to calculate the energy levels and transition parameters, it is based on the multi-configuration Dirac-Hartree-Fock (MCDHF) method. The theoretical basis of this approach has been discussed in many reports [13-16]. So we gave a brief summarization of the MCDHF method in this paper. For a given $N$ electronic system, the configuration state functions (CSFs) $\langle Г Р Ј M|$ are composed by a liner combination of Slater determinants of the single electron Dirac orbitals. The atomic state functions (ASFs) $\left|\gamma_{\mathrm{r}} P J M\right\rangle$ are obtained by a linear combination of CSFs with the same magnetic quantum number $\mathrm{M}$, parity and total angular momentum $\mathrm{J}$ :

$$
\left\langle\Gamma P J M\left|=\sum_{r=1}^{n_{c}} c_{r \Gamma}\right| \gamma_{r} P J M\right\rangle
$$


Where $c_{r \Gamma}$ is the configuration mixing coefficient for each state $\gamma$. The Dirac-Coulomb Hamiltonian of the system is

$$
\hat{H}^{D C}=\sum_{i=1}^{N} \hat{H}_{i}+\sum_{i<j}^{N}\left|r_{i}-r_{j}\right|^{-1}
$$

Where the second item is the coulomb interaction between two electrons in an instant, the first $\widehat{H}_{i}$ is the Hamiltonian of the single electron $i$ expressed in

$$
\hat{H}_{i}=c \alpha \cdot \hat{P}_{i}+(\beta-1) c^{2}+V_{n u c .}\left(r_{i}\right)
$$

So the Dirac-Coulomb Hamiltonian can be given in

$$
\hat{H}^{D C}=\sum_{I=1}^{N}\left[c \alpha \cdot \hat{P}_{i}+(\beta-1) c^{2}+V_{\text {nuс. }}\left(r_{i}\right)\right]_{i}+\sum_{i<j}^{N}\left|r_{i}-r_{j}\right|^{-1}
$$

Where $\widehat{P}_{l}$ is momentum operator, $V_{\text {nuc. }}\left(r_{i}\right)$ is Nuclear potential field, $\alpha$ and $\beta$ are $4 \times 4$ Dirac matrices. The $V$ is the monopole part of the electron-nucleus Coulomb interaction. In the relativistic self-consistent field procedure, the Configuration mixing coefficients and radial parts of the Dirac orbitals were optimized to self-consistency. Some important high order effects should be considered in the process of calculation. In this work, the transverse photon interaction (Breit) and the quantum electrodynamics (QED) corrections including self-energy and vacuum polarization were considered as perturbative corrections. The wavelength of the transition is obtained according to

$$
E=h c / \lambda
$$

The lifetime of energy levels is the reciprocal of the sum of all transition rates from this level to other low levels:

$$
\tau_{i}=\frac{1}{\sum_{j} A_{i j}}
$$

In the MCDHF theory, the configuration state functions (CSFs) are generated by restrictive active space methods, it means that electrons are limited to be active between specified orbitals. We do this by exciting electrons from the reference configurations to a set of orbitals called the active set (AS). Then we expand the configuration space by increasing the principal quantum number systematically.

In this work, we calculated the energy levels of Sb-like $\mathrm{Cs}^{4+}, \mathrm{Ba}^{5+}, \mathrm{La}^{6+}, \mathrm{Ce}^{7+}$, $\mathrm{Pr}^{8+}, \mathrm{Nd}^{9+}, \mathrm{Pm}^{10+}$ and $\mathrm{Sm}^{11+}$ ions and the transition rates, wavelength and lifetimes of $\mathrm{La}^{6+}, \mathrm{Ce}^{7+}, \operatorname{Pr}^{8+}, \mathrm{Nd}^{9+}, \mathrm{Pm}^{10+}$ and $\mathrm{Sm}^{11+}$. The ground state of $\mathrm{Sb} \mathrm{I}$ is $1 s^{2} 2 s^{2} 2 p^{6} 3 s^{2} 3 p^{6} 3 d^{10} 4 s^{2} 4 p^{6} 4 d^{10} 5 s^{2} 5 p^{3}$, we included valence-valence correlations by keeping the core $1 s^{2} 2 s^{2} 2 p^{6} 3 s^{2} 3 p^{6} 3 d^{10} 4 s^{2} 4 p^{6} 4 d^{10}$ closed and common to all calculations. The CSFs are generated by triplet substitutions from the reference configuration to an active set of orbitals. In the first step of the calculation, we set the ground state as the reference configuration and the $5 \mathrm{~s} 、 5 \mathrm{p}$ were active. And then we 
added the $5 \mathrm{~d} 、 5 \mathrm{f}$ and $5 \mathrm{~g}$ shells to our active set. For the $n=6$ orbitals, we still use a complete VV space approach and add the $n=6$ (with $l \leqslant 4$ ) orbitals to the active set.

\section{Results and discussion}

\subsection{Energy levels}

As mentioned earlier, the noteworthy cases are those that occur in HCIs with $Z_{\text {ion }}$ $\geqslant 5$ and the crossing of Sb-like ions occurs at a relatively higher degree of ionization [11]. Hence we infer that the level crossing may happen in $\mathrm{Ce}^{7+}$ or $\operatorname{Pr}^{8+}$, but there are few reference about these ions so we calculate the $\mathrm{Cs}^{4+}$ and $\mathrm{Ba}^{5+}$ to verify the veracity of our result. Table I presents the energy levels of Sb-like isoelectronic ions, along with NIST experimental values for $\mathrm{Cs}^{4+}$ and $\mathrm{Ba}^{5+}$. The energy levels without any corrections are presented in the column E, the contributions of Breit and QED corrections are given separately in columns Breit and QED. The final energy levels are exhibited in the column Final. From the results we know that the contribution of Breit correction is significant while the influence of QED correction is neglected for it is smaller than $0.1 \%$. The relative error with experiment is given in the column Diff. The atomic data for Sb-like isoelectronic ions is fragmentary, only $\mathrm{Cs}^{4+}$ and $\mathrm{Ba}^{5+}$ have been researched before experimentally [16], all of the relative error are smaller than $2 \%$, so we judge that our results are in good agreement with data from NIST. Because all calculated ions belong to $\mathrm{Sb}$-like isoelectronic ions, thus we can indicate that our calculations about $\mathrm{La}^{6+}, \mathrm{Ce}^{7+}, \mathrm{Pr}^{8+}, \mathrm{Nd}^{9+}, \mathrm{Pm}^{10+}$ and $\mathrm{Sm}^{11+}$ ions are credible.

The $5 p-4 f$ level crossing in the $\mathrm{Sb}$ isoelectronic sequence occurs for $\mathrm{Pr}^{8+}-\mathrm{Sm}^{11+}$ ions, the order of these levels in previous ions like $\mathrm{Cs}^{4+}, \mathrm{Ba}^{5+}, \mathrm{La}^{6+}$ and $\mathrm{Ce}^{7+}$ is $5 p^{3}$, $4 f 5 p^{2}, 4 f^{2} 5 p$ and $4 f^{3}$, there are only $5 p^{3}$ levels in table $\mathrm{I}$ for $\mathrm{Cs}^{4+}, \mathrm{Ba}^{5+}, \mathrm{La}^{6+}$ and $\mathrm{Ce}^{7+}$ because the energy difference between $5 p^{3}$ and $4 f 5 p^{2}$ is too large so we just present several lowest levels. The order of these levels changes from $\operatorname{Pr}^{8+}$, the ground state is still $5 p^{3}$ but other low-lying levels belonging to either $4 f 5 p^{2}$ or $5 p^{3}$ configurations cause the energy of some $4 f$ orbitals are smaller than some $5 p$ orbitals so that these $4 f 5 p^{2}$ levels go to front of some $5 p^{3}$ levels, as the $4 f 5 p^{24} \mathrm{G}_{5 / 2}$ and $5 p^{32} \mathrm{D}_{3 / 2}$ in Table I. For $\mathrm{Nd}^{9+}$, the ground state changes to $4 f 5 p^{2}$ with other low-lying levels belonging to either $4 f 5 p^{2}$ or $4 f^{2} 5 p$ configurations. And the $4 f^{2} 5 p$ becomes the ground state for $\mathrm{Pm}^{10+}$ with other low-lying levels belonging to either $4 f^{3}$ or $4 f^{2} 5 p$ configurations. For $\mathrm{Sm}^{11+}$, the ground state and several lowest levels are all $4 f^{3}$, the order of these levels changes to $4 f^{3}, 4 f^{2} 5 p, 4 f 5 p^{2}$ and $5 p^{3}$. The energy differences between $5 p^{3}$ and $4 f 5 p^{2}$, $4 f 5 p^{2}$ and $4 f^{2} 5 p$ or $4 f^{2} 5 p$ and $4 f^{3}$ are getting smaller and smaller near the level crossing. Further, the energy differences between the ground state and several low excited states are small enough to be excited by lasers.

Table I. The level energies (in $\mathrm{cm}^{-1}$ ) of Sb-like $\mathrm{Cs}^{4+}, \mathrm{Ba}^{5+}, \mathrm{La}^{6+}, \mathrm{Ce}^{7+}, \mathrm{Pr}^{8+}, \mathrm{Nd}^{9+}, \mathrm{Pm}^{10+}$ ions, the energy levels without any corrections calculated by MCDHF are presented in columns E, the 
infiuence of Breit and QED corrections are given respectively in columns Breit and QED, the final energy levels taking account of Breit corrections are presented in the column Final. The relative error with experiment is given in the column Diff (in \%).

\begin{tabular}{|c|c|c|c|c|c|c|c|c|}
\hline & CSFs & Term & $\mathrm{E}$ & Breit & QED & Final & NIST $^{\mathrm{a}}$ & Diff.\% \\
\hline \multirow[t]{5}{*}{$\mathrm{Cs}^{4+}$} & $5 p^{3}$ & ${ }^{4} \mathrm{~S}_{3 / 2}$ & 0 & 0 & 0 & 0 & 0 & 0 \\
\hline & $5 p^{3}$ & ${ }^{2} \mathrm{D}_{3 / 2}$ & 15392 & -73 & 8 & 15319 & 15077 & 1.6 \\
\hline & $5 p^{3}$ & ${ }^{2} \mathrm{D}_{5 / 2}$ & 20658 & -137 & 13 & 20521 & 20373 & 0.7 \\
\hline & $5 p^{3}$ & ${ }^{2} \mathrm{P}_{1 / 2}$ & 32692 & -140 & 9 & 32552 & 31951 & 1.8 \\
\hline & $5 p^{3}$ & ${ }^{2} \mathrm{P}_{3 / 2}$ & 42571 & -309 & 28 & 42261 & 42273 & 0.02 \\
\hline \multirow[t]{5}{*}{$\mathrm{Ba}^{5+}$} & $5 p^{3}$ & ${ }^{4} \mathrm{~S}_{3 / 2}$ & 0 & 0 & 0 & 0 & 0 & 0 \\
\hline & $5 p^{3}$ & ${ }^{2} \mathrm{D}_{3 / 2}$ & 17493 & -117 & 11 & 17376 & 17260 & 0.6 \\
\hline & $5 p^{3}$ & ${ }^{2} \mathrm{D}_{5 / 2}$ & 23812 & -182 & 18 & 23630 & 23547 & 0.3 \\
\hline & $5 p^{3}$ & ${ }^{2} \mathrm{P}_{1 / 2}$ & 36959 & -184 & 14 & 36775 & 36155 & 1.7 \\
\hline & $5 p^{3}$ & ${ }^{2} \mathrm{P}_{3 / 2}$ & 49934 & -397 & 37 & 49537 & 49621 & 0.1 \\
\hline \multirow[t]{5}{*}{$\mathrm{La}^{6+}$} & $5 p^{3}$ & ${ }^{4} \mathrm{~S}_{3 / 2}$ & 0 & 0 & 0 & 0 & & \\
\hline & $5 p^{3}$ & ${ }^{2} \mathrm{D}_{3 / 2}$ & 18518 & -128 & 14 & 18390 & & \\
\hline & $5 p^{3}$ & ${ }^{2} \mathrm{D}_{5 / 2}$ & 25256 & -190 & 20 & 25066 & & \\
\hline & $5 p^{3}$ & ${ }^{2} \mathrm{P}_{1 / 2}$ & 38741 & -196 & 20 & 38545 & & \\
\hline & $5 p^{3}$ & ${ }^{2} \mathrm{P}_{3 / 2}$ & 53392 & -416 & 46 & 52976 & & \\
\hline \multirow[t]{5}{*}{$\mathrm{Ce}^{7+}$} & $5 p^{3}$ & ${ }^{4} \mathrm{~S}_{3 / 2}$ & 0 & 0 & 0 & 0 & & \\
\hline & $5 p^{3}$ & ${ }^{2} \mathrm{D}_{3 / 2}$ & 22866 & -252 & 25 & 22614 & & \\
\hline & $5 p^{3}$ & ${ }^{2} \mathrm{D}_{5 / 2}$ & 30561 & -324 & 30 & 30237 & & \\
\hline & $5 p^{3}$ & ${ }^{2} \mathrm{P}_{1 / 2}$ & 46632 & -307 & 26 & 46325 & & \\
\hline & $5 p^{3}$ & ${ }^{2} \mathrm{P}_{3 / 2}$ & 66723 & -637 & 53 & 66086 & & \\
\hline \multirow[t]{5}{*}{$\operatorname{Pr}^{8+}$} & $5 p^{3}$ & ${ }^{4} \mathrm{~S}_{3 / 2}$ & 0 & 0 & 0 & 0 & & \\
\hline & $4 \mathrm{f} 5 \mathrm{p}^{2}$ & ${ }^{4} \mathrm{G}_{5 / 2}$ & 18946 & -560 & -36 & 18386 & & \\
\hline & $5 p^{3}$ & ${ }^{2} \mathrm{D}_{3 / 2}$ & 24752 & -404 & 32 & 24348 & & \\
\hline & $4 f 5 p^{2}$ & ${ }^{4} \mathrm{D}_{7 / 2}$ & 25847 & -862 & -42 & 24985 & & \\
\hline & $5 p^{3}$ & ${ }^{2} \mathrm{D}_{5 / 2}$ & 32343 & -591 & 18 & 31751 & & \\
\hline \multirow[t]{2}{*}{$\mathrm{Nd}^{9+}$} & $4 \mathrm{f} 5 \mathrm{p}^{2}$ & ${ }^{4} \mathrm{G}_{5 / 2}$ & 0 & 0 & 0 & 0 & & \\
\hline & $4 \mathrm{f} 5 \mathrm{p}^{2}$ & ${ }^{4} \mathrm{D}_{7 / 2}$ & 6513 & -345 & 3 & 6168 & & \\
\hline
\end{tabular}




\begin{tabular}{|c|c|c|c|c|c|c|}
\hline & $4 f 5 p^{2}$ & ${ }^{2} \mathrm{G}_{7 / 2}$ & 24389 & -749 & 24 & 23640 \\
\hline & $4 f 5 p^{2}$ & ${ }^{4} \mathrm{G}_{5 / 2}$ & 27102 & -459 & 47 & 26643 \\
\hline & $4 f^{2} 5 p$ & ${ }^{2} \mathrm{D}_{3 / 2}$ & 29973 & -611 & 30 & 29362 \\
\hline & $4 f 5 p^{2}$ & ${ }^{4} \mathrm{G}_{7 / 2}$ & 31113 & -785 & 37 & 30328 \\
\hline & $4 f^{2} 5 p$ & ${ }^{4} \mathrm{G}_{5 / 2}$ & 32348 & -957 & 6 & 31391 \\
\hline & $4 f 5 p^{2}$ & ${ }^{4} \mathrm{~F}_{3 / 2}$ & 34980 & -654 & 31 & 34326 \\
\hline & $5 p^{3}$ & ${ }^{4} \mathrm{~S}_{3 / 2}$ & 40829 & -61 & 45 & 40768 \\
\hline \multirow[t]{10}{*}{$\mathrm{Pm}^{10+}$} & $4 f^{2} 5 p$ & ${ }^{4} \mathrm{I}_{9 / 2}$ & 0 & 0 & 0 & 0 \\
\hline & $4 f^{3}$ & ${ }^{4} \mathrm{I}_{9 / 2}$ & 6262 & -966 & -13 & 5296 \\
\hline & $4 f^{2} 5 p$ & ${ }^{4} \mathrm{H}_{7 / 2}$ & 5612 & -19 & 5 & 5593 \\
\hline & $4 f^{3}$ & ${ }^{4} \mathrm{I}_{11 / 2}$ & 6753 & -544 & 2 & 6209 \\
\hline & $4 f^{2} 5 p$ & ${ }^{4} \mathrm{H}_{9 / 2}$ & 7792 & -321 & -5 & 7471 \\
\hline & $4 f^{2} 5 p$ & ${ }^{4} \mathrm{I}_{11 / 2}$ & 9115 & -1104 & -9 & 8011 \\
\hline & $4 f^{2} 5 p$ & ${ }^{4} \mathrm{G}_{5 / 2}$ & 9438 & 11 & 2 & 9449 \\
\hline & $4 f^{2} 5 p$ & ${ }^{2} \mathrm{D}_{3 / 2}$ & 11706 & -26 & 3 & 11680 \\
\hline & $4 \mathrm{f} 5 \mathrm{p}^{2}$ & ${ }^{4} \mathrm{G}_{5 / 2}$ & 73744 & 682 & 63 & 74426 \\
\hline & $5 p^{3}$ & ${ }^{4} S_{3 / 2}$ & 163424 & 1929 & 75 & 165353 \\
\hline \multirow[t]{9}{*}{$\mathrm{Sm}^{11+}$} & $4 f^{3}$ & ${ }^{4} \mathrm{I}_{9 / 2}$ & 0 & 0 & 0 & 0 \\
\hline & $4 f^{3}$ & ${ }^{4} \mathrm{I}_{11 / 2}$ & 3397 & -337 & 3 & 3063 \\
\hline & $4 f^{3}$ & ${ }^{4} \mathrm{I}_{13 / 2}$ & 6983 & -692 & 4 & 6296 \\
\hline & $4 f^{3}$ & ${ }^{4} \mathrm{I}_{15 / 2}$ & 10679 & -1051 & 6 & 9633 \\
\hline & $4 f^{3}$ & ${ }^{4} \mathrm{~F}_{3 / 2}$ & 19834 & 62 & 1 & 19867 \\
\hline & $4 f^{3}$ & ${ }^{4} \mathrm{~F}_{5 / 2}$ & 21767 & -189 & 2 & 21578 \\
\hline & $4 f^{2} 5 p$ & ${ }^{2} \mathrm{D}_{3 / 2}$ & 71903 & -1208 & 8 & 73111 \\
\hline & $4 f 5 p^{2}$ & ${ }^{4} \mathrm{G}_{5 / 2}$ & 171337 & 229 & 77 & 171566 \\
\hline & $5 p^{3}$ & ${ }^{4} \mathrm{~S}_{3 / 2}$ & 371140 & 3073 & 97 & 374213 \\
\hline
\end{tabular}

aValues from Ref. [13]

Table II. Transition energies $\Delta E$ (in $\mathrm{cm}^{-1}$ ), transition rates $A$ (in $\mathrm{s}^{-1}$ ), wavelengths $\lambda$ (in $\mathrm{nm}$ ) and lifetimes $\tau$ (in sec) in Sb-like ions. All of these are obtained from final energy values given by Table I. The numbers in brackets represent powers of 10 . 


\begin{tabular}{|c|c|c|c|c|c|c|c|}
\hline Ion & Upper & lower & & $\Delta \mathrm{E}$ & $\lambda$ & A & $\tau$ \\
\hline \multirow[t]{4}{*}{$\mathrm{La}^{6+}$} & $5 p^{3}{ }^{2} D_{3 / 2}$ & $5 p^{32} S_{3 / 2}$ & M1 & 18390 & 544.2 & $9.12287[+01]$ & $1.1[-02]$ \\
\hline & $5 p^{32} D_{5 / 2}$ & $5 p^{32} S_{3 / 2}$ & M1 & 25066 & 399.2 & $1.36860[+01]$ & $5.9[-02]$ \\
\hline & & & E2 & 25066 & 399.2 & 1.02636 & \\
\hline & & $5 p^{32} D_{3 / 2}$ & M1 & 6676 & 1499 & 2.19404 & \\
\hline \multirow[t]{9}{*}{$\mathrm{Ce}^{7+}$} & $5 p^{32} D_{3 / 2}$ & $5 p^{32} S_{3 / 2}$ & M1 & 22614 & 442.6 & $2.16603[+02]$ & 0.005 \\
\hline & $5 p^{32} D_{5 / 2}$ & $5 p^{32} S_{3 / 2}$ & E2 & 30237 & 331 & 1.90040 & 0.024 \\
\hline & & & M1 & 30237 & 331 & $3.65461[+01]$ & \\
\hline & & $5 p^{32} D_{3 / 2}$ & M1 & 7623 & 1312.7 & 3.029670 & \\
\hline & $5 p^{32} P_{1 / 2}$ & $5 p^{32} S_{3 / 2}$ & E2 & 46325 & 216 & 8.71302 & 0.002 \\
\hline & & & M1 & 46325 & 216 & $4.36211[+02]$ & \\
\hline & & $5 p^{32} D_{3 / 2}$ & E2 & 23711 & 422 & 2.02959 & \\
\hline & & & M1 & 23711 & 422 & $1.90667[+01]$ & \\
\hline & & $5 p^{32} D_{5 / 2}$ & E2 & 16088 & 6220.01 & $3.17776[-01]$ & \\
\hline \multirow[t]{6}{*}{$\operatorname{Pr}^{8+}$} & $4 f 5 p^{24} G_{5 / 2}$ & $5 p^{34} S_{3 / 2}$ & M1 & 18386 & 544.27 & $3.89334[+01]$ & $2.57[-02]$ \\
\hline & $5 p^{3}{ }^{2} D_{3 / 2}$ & $5 p^{34} S_{3 / 2}$ & E2 & 24348 & 411 & $2.61488[-01]$ & $3.3[-03]$ \\
\hline & & $5 p^{34} S_{3 / 2}$ & M1 & 24348 & 411 & $3.02414[+02]$ & \\
\hline & & $4 f 5 p^{24} G_{5 / 2}$ & E2 & 5962 & 1678.45 & 6.21771]-06] & \\
\hline & $4 f 5 p^{24} D_{7 / 2}$ & $5 p^{34} S_{3 / 2}$ & E2 & 24985 & 400.52 & $1.48869[-01]$ & $2.48[-01]$ \\
\hline & & $4 f 5 p^{24} G_{5 / 2}$ & M1 & 6599 & 1516.43 & $3.88650[+00]$ & \\
\hline \multirow[t]{10}{*}{$\mathrm{Nd}^{9+}$} & $4 f 5 p^{24} D_{7 / 2}$ & $4 f 5 p^{24} \mathrm{G}_{5 / 2}$ & M1 & 6168 & 1622.39 & $3.58614[+00]$ & $2.79[-01]$ \\
\hline & $4 f 5 p^{22} G_{7 / 2}$ & $4 f 5 p^{24} G_{5 / 2}$ & E2 & 23640 & 423.30 & $3.15546[-03]$ & $2.75[-02]$ \\
\hline & & $4 f 5 p^{24} G_{5 / 2}$ & M1 & 23640 & 423.30 & $3.14261[+01]$ & \\
\hline & & $4 \mathrm{f} 5 \mathrm{p}^{24} \mathrm{D}_{7 / 2}$ & E2 & 18113 & 552.47 & $5.09213[-03]$ & \\
\hline & & $4 \mathrm{f} 5 \mathrm{p}^{24} \mathrm{D}_{7 / 2}$ & M1 & 18113 & 552.47 & $4.88835[+00]$ & \\
\hline & $4 f 5 p^{22} G_{5 / 2}$ & $4 f 5 p^{2}{ }^{4} G_{5 / 2}$ & M1 & 26643 & 375.59 & $2.91850[+02]$ & $3.37[-03]$ \\
\hline & & $4 f 5 p^{2}{ }^{4} D_{7 / 2}$ & M1 & 20474 & 488.76 & $4.25268[+00]$ & \\
\hline & & $4 \mathrm{f} 5 \mathrm{p}^{22} \mathrm{G}_{7 / 2}$ & M1 & 3002 & 3333.42 & $9.82796[-02]$ & \\
\hline & $4 f^{2} 5 p^{2} D_{3 / 2}$ & $4 f 5 p^{2 ~}{ }^{4} G_{5 / 2}$ & E2 & 29362 & 340.81 & $1.34959[-02]$ & $1.13[-02]$ \\
\hline & & $4 f 5 p^{2}{ }^{4} G_{5 / 2}$ & M1 & 29362 & 340.81 & $8.82127[+01]$ & \\
\hline
\end{tabular}




\begin{tabular}{|c|c|c|c|c|c|c|c|}
\hline & & $4 f 5 p^{24} D_{7 / 2}$ & E2 & 23194 & 431.44 & $2.61054[-02]$ & \\
\hline & & $4 f 5 p^{22} G_{5 / 2}$ & M1 & 2720 & 3679.01 & $1.40089[-01]$ & \\
\hline \multirow[t]{10}{*}{$\mathrm{Pm}^{10+}$} & $4 \mathrm{f}^{34} \mathrm{I}_{9 / 2}$ & $4 f^{2} 5 p^{4} I_{9 / 2}$ & M1 & 5296 & 1889.52 & $2.84553[-02]$ & 35.14 \\
\hline & $4 f^{2} 5 p^{4} \mathrm{H}_{7 / 2}$ & $4 f^{2} 5 p^{4} I_{9 / 2}$ & M1 & 5593 & 1789.19 & $2.01045[-01]$ & 4.97 \\
\hline & $4 f^{3} I_{11 / 2}$ & $4 f^{2} 5 p^{4} I_{9 / 2}$ & M1 & 6209 & 1611.68 & $5.81502[+00]$ & 0.17 \\
\hline & $4 f^{2} 5 p^{4} H_{9 / 2}$ & $4 f^{2} 5 p^{4} I_{9 / 2}$ & M1 & 7471 & 1339.43 & $1.94216[-01]$ & 2.66 \\
\hline & & $4 \mathrm{f}^{34} \mathrm{I}_{9 / 2}$ & M1 & 2175 & 4600.88 & $2.69243[-03]$ & \\
\hline & & $4 \mathrm{f}^{2} 5 \mathrm{p}^{4} \mathrm{H}_{7 / 2}$ & M1 & 1878 & 5328.5 & $1.70346[-01]$ & \\
\hline & & $4 \mathrm{f}^{3} \mathrm{I}_{11 / 2}$ & M1 & 1262 & 7929.41 & $8.90877[-03]$ & \\
\hline & $4 \mathrm{f}^{34} \mathrm{I}_{11 / 2}$ & $4 f^{2} 5 p^{4} I_{9 / 2}$ & M1 & 8011 & 1249.15 & $1.39465[+00]$ & 0.48 \\
\hline & & $4 \mathrm{f}^{3}{ }^{4} \mathrm{I}_{9 / 2}$ & M1 & 2714 & 3687.15 & $6.73103[-01]$ & \\
\hline & & $4 f^{3} I_{11 / 2}$ & M1 & 1802 & 5553.23 & $2.37105[-03]$ & \\
\hline \multirow[t]{5}{*}{$\mathrm{Sm}^{11+}$} & $4 f^{3} I_{11 / 2}$ & $4 \mathrm{f}^{34} \mathrm{I}_{9 / 2}$ & M1 & 3063 & 3267 & $1.20119[+00]$ & 0.83 \\
\hline & $4 f^{3}{ }^{4} I_{13 / 2}$ & $4 \mathrm{f}^{3} \mathrm{I}_{9 / 2}$ & M3 & 6296 & 1589.41 & $4.57536[-23]$ & 0.61 \\
\hline & & $4 f^{3}{ }^{4} I_{11 / 2}$ & M1 & 3233 & 3095.24 & $1.62660[+00]$ & \\
\hline & $4 \mathrm{f}^{3} \mathrm{I}_{15 / 2}$ & $4 \mathrm{f}^{34} \mathrm{I}_{9 / 2}$ & M3 & 9633 & 1038.82 & $3.21145[-22]$ & 0.84 \\
\hline & & $4 f^{3}{ }^{4} I_{13 / 2}$ & M1 & 3337 & 2998.78 & $1.19054[+00]$ & \\
\hline
\end{tabular}

\subsection{Transition parameters}

Because transitions near level crossings in HCIs can provide higher sensitivity to $\alpha$ variation than others $[9,10]$, we focused on $\mathrm{La}^{6+}, \mathrm{Ce}^{7+}, \mathrm{Pr}^{8+}, \mathrm{Nd}^{9+}, \mathrm{Pm}^{10+}$ and $\mathrm{Sm}^{11+}$ ions according to our results. The transition rates $A$ (in s${ }^{-1}$ ), wavelengths $\lambda$ (in $\mathrm{nm}$ ) and lifetimes $\tau$ (in sec) are showed in Table II. The numbers in brackets represent powers of 10. There is no any reference to be compared, but we can use the energy reference to estimate the uncertainties of the transition parameters calculations. It is remarkable that these ions have strong electric quadrupole $(E 2)$ or magnetic dipole $(M 1)$ transitions hence they have long-lived metastable states. Especially $\mathrm{Pm}^{10+}$ and $\mathrm{Sm}^{1{ }^{1+}}$, which have metastable states with very long lifetimes. For $\mathrm{Pm}^{10+}$, the lowest metastable state $4 f^{3}{ }_{1} \mathrm{I}_{9 / 2}$ has a very long lifetime with $35.14 \mathrm{~s}$ and its wavelength to the ground state is $1889.52 \mathrm{~nm}$ which can be excited by existing laser. The next two levels $4 f^{2} 5 p^{4} \mathrm{H}_{7 / 2}$ and $4 f^{34} \mathrm{I}_{11 / 2}$ also have optical transitions to the ground state and they have long lifetimes with $4.97 \mathrm{~s}$ and $0.17 \mathrm{~s}$. The low-lying levels of $\mathrm{Nd}^{13+}$ ion, transitions and the radiative lifetimes are shown in Fig. 1. The transitions between some low excited states are ignored because that the transition rates are too small. It is 
more intuitive to show the transitions between these low-lying levels and the long lifetimes.

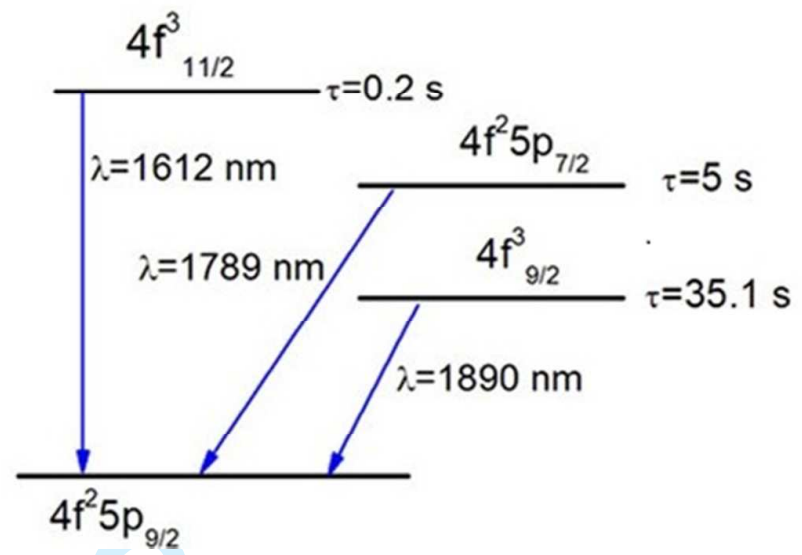

FIG. 1. Transitions(color online), energy levels and radiative lifetimes of low-lying levels of Sb-like $\mathrm{Pm}^{10+}$.

The several low-lying levels, transitions and our calculation of the radiative lifetimes of $\mathrm{Sm}^{1{ }^{+}}$ion are given in Fig. 2. The lowest metastable state $4 f^{3} \mathrm{I}_{11 / 2}$ for $\mathrm{Sm}^{11+}$ have the strong M1 transition in the optical range to the ground state with a long lifetime $0.83 \mathrm{~s}$, the next two low excited states $4 f^{34} \mathrm{I}_{13 / 2}$ and $4 f^{34} \mathrm{I}_{15 / 2}$ also have appropriate transition wavelength to the ground state and long lifetimes but the transitions to the ground state are too weak. Compared with $\mathrm{Nd}^{13+}$ ions, the lifetimes of the $\mathrm{Sm}^{13+}$ levels are smaller (less than $1 \mathrm{~s}$ ), whereas the advantage of smaller lifetimes is that it can make locate the transitions simpler, hence it can be used as a development object.

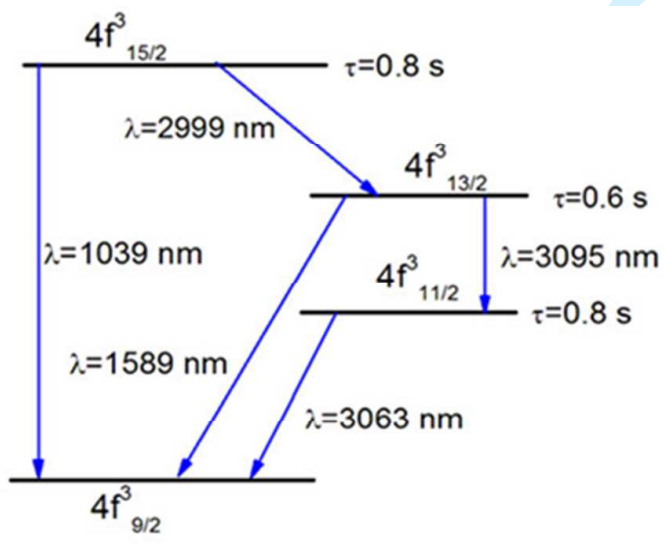

FIG.2. Transitions(color online), energy levels and radiative lifetimes of low-lying levels of Sb-like $\mathrm{Sm}^{11+}$.

\section{Summary}

In this work, the atomic properties and transition parameters of Sb-like $\mathrm{Cs}^{4+}$, $\mathrm{Ba}^{5+}, \mathrm{La}^{6+}, \mathrm{Ce}^{7+}, \mathrm{Pr}^{8+}, \mathrm{Nd}^{9+}, \mathrm{Pm}^{10+}$ and $\mathrm{Sm}^{11+}$ ions were performed by GRASP2K which based on multi-configuration relativity Dirac-Hartree-Fock (MCDHF) method. 
The Breit and QED correlations were considered in the calculation of energy levels, only the contribution of Breit correlation was taken into account in final results because that it is more dominant than QED. Our results about energy levels of $\mathrm{Cs}^{4+}$ and $\mathrm{Ba}^{5+}$ agreed well with the previous experimental value. The level crossing of $\mathrm{Sb}$-like isoelectronic ions occurs from $\mathrm{Pr}^{8+}$ to $\mathrm{Sm}^{11+}$. The transition energies from low excited states to the ground state are relatively small near the level crossing. Particularly $\mathrm{Pm}^{10+}$ and $\mathrm{Sm}^{11+}$ which the suitable transitions within the optical range, and they have long-lived metastable states. These highly charged ions are very important for researches of variation of $a$ and the atomic clocks. The researches about Sb-like $\mathrm{La}^{6+}, \mathrm{Ce}^{7+}, \mathrm{Pr}^{8+}, \mathrm{Nd}^{9+}, \mathrm{Pm}^{10+}$ and $\mathrm{Sm}^{11+}$ ions are lacking and our results can add the database and provide a reference for further research.

\section{Author contribution statement}

The authors express genuine gratitude to P .Jönsson for providing the GRASP2k program for free. This work was supported by the National Nature Science Foundation of China (Grant No. 11474208).

\section{References}

[1] T. Rosenband, D. B. Hume, P. O. Schmidt, C. W. Chou,A. Brusch, L. Lorini, W. H. Oskay, R. E. Drullinger, T. M. Fortier, J. E. Stalnaker et a., Science 319, 1808 (2008)

[2] C. W. Chou, D. B. Hume, J. C. J. Koelemeij, D. J. Wineland, and T. Rosenband, Phys. Rev. Lett. 104, 070802 (2010)

[3] G.K. Campbell et al., Metrologia 45, 539 (2008).

[4] Derevianko, V. A. Dzuba and V. V. Flambaum, Phys. Rev. Lett. 109, 180801 (2012)

[5] V. A. Dzuba, A. Derevianko, and V. V. Flambaum, Phys. Rev. A 86, 054502 (2012)

[6] M. S. Safronova, V. A. Dzuba, V. V. Flambaum. Phys. Rev. A. 90.042513 (2014)

[7] M. S. Safronova,V. A. Dzuba, V. V. Flambaum, Phys. Rev. A. 90.052509 (2014)

[8] J. C. Berengut, V. A. Dzuba, V. N. Huntemann, M. Okhapkin, B. Lipphardt, S. Weyers, Chr. Tamm, and E. Peik. Phys. Rev. Lett.108.090801. (2012)

[9] J. C. Berengut, V. A. Dzuba, and V. V. Flambaum, Phys. Rev. Lett. 105, 120801 (2010)

[10] J. C. Berengut, V. A. Dzuba, V. V. Flambaum, and A. Ong, Phys. Rev. Lett. 106, 210802 (2011)

[11] J. C. Berengut, V. A. Dzuba,V. Flambaum, and A. Ong, Phys. Rev. A. 86.022517 (2012)

[12] Kramida, A., Ralchenko, Yu., Reader, J. and NIST ASD Team (2016). NIST Atomic Spectra Database (version 5.4). Available: http://physics.nist.gov/asd. National Institute of Standards and Technology, Gaithersburg, MD.

[13] J. Olsen, M. R. Godefroid, P. A. Jonsson, P. A. Malmquist, andF. C. Froese, Phys. Rev. E 52, 4499 (1995).

[14] L. H. Hao, and G. Jiang, Phys. Rev .A. 83.012511 (2011)

[15] L. H. Hao, G. Jiang, and H. J. Hou, Phys. Rev. A. 81, 022502 (2010) 
[16] G. J. Bian, G. Jiang, Q. P.Fan and F. Hu, Phys. Scr. 90.015403 (2015) 